\title{
The Role of Surface Feature Continuity in Object-based Inhibition of Return
}

\author{
A. Caglar Tas, \\ University of lowa, IA, USA \\ Michael D. Dodd, and \\ University of Nebraska-Lincoln, NE, USA
}

\section{Andrew Hollingworth}

University of lowa, IA, USA

\begin{abstract}
The contribution of surface feature continuity to object-based inhibition of return (IOR) was tested in three experiments. Participants executed a saccade to a previously fixated or unfixated colored disk after the object had moved to a new location. Object-based IOR was observed as lengthened saccade latency to a previously fixated object. The consistency of surface feature (color) and spatiotemporal information was manipulated to examine the feature used to define the persisting objects to which inhibition is assigned. If the two objects traded colors during motion, objectbased IOR was reliably reduced (Experiment 2), suggesting a role for surface feature properties in defining the objects of object-based IOR. However, if the two objects changed to new colors during motion, object-based IOR was preserved (Experiment 1), and color consistency was not sufficient to support object continuity across a salient spatiotemporal discontinuity (Experiment 3). These results suggest that surface feature consistency plays a significant role in defining object persistence for the purpose of IOR, although surface features may be weighted less strongly than spatiotemporal features in this domain.
\end{abstract}

Visual perception is a dynamic process that introduces frequent change and disruption. The distal stimulus often changes (as a result of object motion and other environmental change), the proximal input to vision frequently changes (with shifts in retinal projection created by eye, head, and body movement), and visual input is often disrupted entirely (by events such as saccadic suppression, blinks, and occlusion). Yet, despite the instability of visual input, there is a pressing need to keep track of objects in the world as stable, continuous entities. A dog observed to be snarling at one moment must be perceived at some later point as the same dog in order for knowledge about its temperament to guide intelligent interaction. When searching for a friend among passengers exiting a train, a previously attended stranger must be treated as the same person across movement and disruption to avoid directing attention back to him. To perceive the world as continuous and not generated anew upon each fixation, an object at one retinal location before a saccade must be treated as the same object when it appears at a different retinal location after the saccade. Each of these examples instantiates the correspondence problem in vision: How does the visual system establish the mapping, or correspondence, between individual objects across change and disruption so as to treat environmental objects as stable, persisting entities?

Correspondence should be addressed to A. Caglar Tas, Department of Psychology, The University of Iowa, 11 Seashore E, Iowa City, IA, USA, 52242-1407. (caglar-tas@uiowa.edu), Phone: 319-335-0304. 
There are two main sources of information that could be used to compute object correspondence. First, correspondence could be computed on the basis of an object's spatiotemporal features. If an object's location over time is consistent with the interpretation of a continuous entity, then object correspondence can be established (e.g. Kahneman, Treisman, \& Gibbs, 1992). For example, if an object appears at a new location in a manner that is consistent with a unique entity traveling on a continuous motion trajectory, it can be perceived as a single object. Or, if an object disappears momentarily behind an occluder, it can be treated as the same object if its post-occlusion position is consistent with its spatiotemporal history. Second, correspondence could be computed on the basis of an object's surface features, such as shape, color, size, and texture. If an object's surface features are continuous across time, change, or disruption, then object correspondence could be established. For example, if an object visible after a saccade shares the shape and color of an object appearing before a saccade, it could be treated as a single, continuous object. Or, if an object's surface features are consistent across a brief occlusion, the object could be interpreted as continuous (e.g., if one's own dog, containing all the surface features known to be associated with that dog, walks behind a tree and then re-emerges, it will plausibly be treated as the same dog regardless of whether it appears from the expected or unexpected side given its motion history).

Despite the availability of multiple cues for computing object correspondence, the dominant view for many years has been that spatiotemporal features of an object are central to correspondence operations and that the surface features of an object are consulted only after correspondence has already been established on the basis of spatiotemporal continuity (Kahneman et al., 1992) or consulted only when spatiotemporal information is unavailable or ambiguous (Flombaum, Scholl, \& Santos, 2009). The evidence cited in favor of this view has been obtained from a range of content areas and experimental paradigms (for a review, see Flombaum et al., 2009), organized by the theoretical framework proposed by Kahneman et al (1992). In the Kahneman et al.'s object-file theory, objects are proposed to be defined by their spatial positions over time, indexed by spatial markers which are content independent (e.g., Pylyshyn, 2000). If an object moves or its representation is disrupted, the marker can bridge the change or gap, and the representation is reassigned to the original object on the basis of its spatiotemporal consistency. An object that is assigned the same spatial marker that it had originally is then treated as functionally continuous. The content associated with the spatial index (such as object surface features) can be retrieved only after spatiotemporal correspondence has been established and thus cannot play a primary role in the initial computation of correspondence.

Empirical evidence supporting the object-file theory comes primarily from the objectreviewing paradigm (Kahneman et al., 1992). In this paradigm, two preview letters are briefly presented in two objects. Then, the objects move to new locations and a test letter appears in one of them. The typical finding is that participants are faster to name the test letter if it appears in the same object as it had previously (referred as 'the object-specific preview benefit'). In a modified version of this paradigm, Mitroff and Alvarez (2007) manipulated both spatiotemporal and surface feature continuity of the objects. In their spatiotemporal condition, the letters appeared in two identical empty boxes, after which the boxes moved to new locations and a letter appeared in one of them (as in the original objectreviewing experiment). The task was to report whether the test letter had appeared in the preview display. In their feature condition, the preview letters appeared in objects with different surface features (e.g., a blue square with a hole and a red circle). The object positions were changed in a single step, eliminating spatiotemporal continuity, followed by the test display. Mitroff and Alvarez found an object-specific preview benefit only for the spatiotemporal condition, suggesting that spatiotemporal information was dominant in object correspondence. Specifically, they suggested that when spatiotemporal continuity is 
ambiguous, surface feature continuity is not sufficient to produce a persisting object representation.

Although spatiotemporal continuity has been regarded as the primary information used in object correspondence, a growing body of evidence demonstrates that surface features are also consulted in correspondence operations. Using a modified version of the Mitroff and Alvarez paradigm, Hollingworth and Franconeri (2009) showed that surface feature continuity can generate an object-specific preview benefit typically found under conditions of spatiotemporal continuity. Participants saw two colored objects which initially contained novel preview shapes for a brief duration as the objects moved behind an occluder. After a brief delay, the occluder was removed, making the two objects visible with a novel test shape in each object. The task was to indicate whether the two test shapes were the same as the preview shapes, regardless of their positions or the color of the objects in which they appeared. A significant object-specific preview benefit was found when the test shapes were present in the original objects as defined by spatiotemporal history. Importantly, a significant object-specific preview benefit was also found when the test shapes were present in the original objects as defined by surface feature history (color). Further, colorconsistency effects were found even when spatiotemporal information was discontinuous, suggesting that color can drive object correspondence even in the presence of conflicting spatiotemporal information. This key finding has been extended by Moore, Stephens, and Hein (2010). In a direct replication of the Mitroff and Alvarez method, Moore et al. found a significant surface-feature consistency effect when the objects were moved, in a single step, to new locations.

There are two limitations, however, inherent in the object reviewing paradigms used to test the relative contributions of spatiotemporal and surface features. First, the performance measure concerns a secondary feature (letters or shapes that appear briefly within the objects) that may be only indirectly associated with the primary objects whose continuity is being manipulated. Second, recent versions of the object-reviewing paradigm have depended on explicit memory for the task-relevant, secondary features of the objects (Hollingworth \& Franconeri, 2009; Mitroff \& Alvarez, 2007; Noles, Scholl, \& Mitroff, 2005)..$^{1}$ Participants are required to remember the letters or shapes that appear within the objects and report, explicitly, whether those features are present in the test display. Such explicit memory for object features may not be entirely characteristic of the type of rapid, online correspondence operations that occur in natural vision (see Mitroff, Scholl, and Wynn, 2005).

In the present study, we measured object correspondence with a more direct paradigm, using a performance measure (saccade latency to an object) that requires direct orienting to the manipulated object itself. In addition, the task did not require explicit memory or conscious report, providing a measure of object correspondence without the requirement to explicitly retain secondary stimuli associated with the manipulated object.

\section{Present Study}

We tested the contribution of each type of information within an object-based inhibition of return (IOR) paradigm. Briefly, inhibition of return is delayed stimulus detection at and/or delayed orienting to a previously attended location (Posner \& Cohen, 1984). Object-based IOR is observed when a scene contains moving stimuli instead of stationary objects. In this

\footnotetext{
${ }^{1}$ The original Kahneman et al. (1992) method did not require explicit memory, as the response (speeded naming of a test letter) could have been generated without memory for the preview letters. However, Kahneman et al. did not test the relative contributions of spatiotemporal and surface features to object persistence.
} 
case, inhibition is assigned to a particular object and can be observed even after the object moves to another location (Tipper, Weaver, Jerreat, \& Burak, 1994). In a standard objectbased IOR paradigm, participants are presented with a display containing multiple objects. Attention is cued to one of the objects and then directed back to the center. The objects then move to occupy new locations. Finally, a target appears in either the cued (i.e. previously attended) or an uncued (i.e. previously unattended) object. The typical finding is that participants are slower to detect the target in the cued object than in an uncued object. Object-based IOR has been found both when the participants were required to shift gaze to the target object (i.e., overt shifts of attention; Abrams \& Dobkin, 1994) and when they were required to fixate the center of the screen throughout the trial (i.e., covert shifts of attention; Tipper et al., 1994). Because this type of inhibition is object-specific, it provides a direct measure of object correspondence. Specifically, a significant object-based IOR effect should only be observed when the visual system treats the previously attended object as the same object across a change in its location. The object-based IOR paradigm does not require the explicit report of object features or report of conscious experience; all that is required is simple detection of an unrelated target (Tipper et al., 1994) or orienting to the target object (Abrams \& Dobkin, 1994). ${ }^{2}$

We developed a modified version of the object-based IOR paradigm (see Figure 1). At the beginning of each trial, participants were instructed to fixate on a center dot. Two colored disks appeared on opposite sides of fixation, separated by $180^{\circ}$. One of the disks was cued (see Methods), and participants were required to execute a saccade to that disk. After fixation of the cued object, the central dot was cued, and participants executed a saccade back to the center of the display. Next, the colored disks moved smoothly to new locations. A small target dot then appeared in either the cued or uncued disk. Participants were instructed to execute a saccade to the target object as quickly as possible. Participants' eye movements were recorded, and saccade latency to the target object was used as the dependent measure, with object-based IOR indicated by slower saccadic reaction times when the target was the previously fixated object compared with the unfixated object.

To probe the contributions of surface feature and spatiotemporal continuity, both sources of information were systematically manipulated in three experiments. In Experiments 1 and 2, we kept spatiotemporal information consistent but changed the objects' surface feature information (i.e., color) while they were moving to new locations. In Experiment 3, surface feature information was kept consistent, but we introduced a spatiotemporal discontinuity by eliminating the linking motion between the objects' original and new positions.

\section{Experiment 1}

In Experiment 1, we examined the effect of surface feature continuity on object correspondence by changing the objects' surface features while they were moving to new locations. For half of the trials, the objects retained their original colors throughout the trial (no change), whereas for the other half, the objects colors were changed to novel colors while moving to new locations (color change). The color change trials introduced a salient change in the surface features of the objects during motion. Moore et al. (2007) found that

\footnotetext{
${ }^{2}$ Studies investigating IOR have provided initial evidence regarding the role of surface features in the duration and magnitude of inhibition. Paul and Tipper (2003) compared IOR for objects which had distinctive features (i.e., objects with different colors and shapes) and objects that had similar features (i.e., gray squares). Although the magnitude of the inhibition was similar for both types of objects, inhibition for distinctive objects lasted longer and was less sensitive to interference from new items. In a similar vein, Morgan and Tipper (2007) manipulated the surface feature similarity of the cued and uncued objects, and found that the magnitude of IOR was reliably higher when cued and uncued objects had exactly the same shape compared to when they were slightly different. Although the above studies demonstrate that surface feature similarity is a significant contributor to IOR, IOR in these cases was probed over multitrial retention intervals depending on long-term memory, and thus do not necessarily inform the computation of online object correspondence.
} 
such a salient surface feature change results in disruption of perception of a single, continuous object, even when the spatiotemporal continuity is consistent with a single object perception. With the same reasoning, it was expected that IOR would not be observed for the color-change trials because the objects would not be treated as the same objects that were present (and attended) at the start of the trial.

\section{Method}

Participants-Twenty-one undergraduate students from the University of Iowa participated for course credit. All participants had normal, non-corrected vision. Data from one participant was excluded from the analyses due to failure to comply with the instruction to consistently generate a saccade to the cued object and then back to the center prior to object motion.

Stimuli and Apparatus-The experimental display consisted of a black fixation dot that subtended $0.6^{\circ}$ of visual angle and two peripheral color disks that subtended $1.64^{\circ}$ and were centered $9.89^{\circ}$ from the fixation disk. The background was a neutral gray (RGB: 148, 148, 148). The peripheral disks appeared at a random location on an imaginary circle, $180^{\circ}$ opposite of each other. Two of the following colors were randomly selected for peripheral disks' initial colors: blue (RGB: 18, 18, 255), green (RGB: 0, 138, 0), red (RGB: 220, 18, 18 ), and orange (RGB: $222,111,0$ ). The target dot subtended $0.2^{\circ}$ (RGB: 148, 148, 148), and appeared at the center of one of the disks.

Stimuli were displayed to the participants on a 17 -in. CRT monitor with a $120 \mathrm{~Hz}$ refresh rate. Eye position was monitored by a video-based, Eyelink 2000 eyetracker sampling at $1000 \mathrm{~Hz}$. The experiment was also displayed on another monitor to give the experimenter real-time feedback and to allow for re-calibrations as necessary. A chin and forehead rest was used to ensure a $70 \mathrm{~cm}$ viewing distance and to minimize head movements. Stimulus presentation was controlled by E-prime software. All images were pre-loaded into graphics memory at the beginning of each trial. The onset of each stimulus was synchronized to the monitor's vertical retrace.

Procedure-The sequence of events for each trial is presented in Figure 1. At the beginning of the experiment, the eyetracker was calibrated with a 9-point calibration procedure, and the calibration was repeated during the experiment when necessary. At the beginning of each trial, participants were instructed to fixate the central, black fixation dot, whereupon the experimenter initiated the trial. Three hundred milliseconds after the start of the trial, the screen was replaced with a preview array consisting of the central fixation dot and two peripheral color disks. After $1000 \mathrm{~ms}$, one of the peripheral disks was cued: It expanded in two steps to $140 \%$ of its original size and contracted in two steps back to its original size. The duration of the cue event was $50 \mathrm{~ms}$. The cued disk was chosen randomly. Participants executed a saccade to the cued object. Four hundred milliseconds after the peripheral cue, the center dot was cued for $300 \mathrm{~ms}$ by turning it white, and participants shifted gaze back to the center. After another $200 \mathrm{~ms}$ of delay, the peripheral disks began rotating $90^{\circ}$ (in polar coordinates) in a clockwise direction which lasted for $250 \mathrm{~ms}$. The motion was completed across 30 frames $\left(8.3 \mathrm{~ms} /\right.$ frame), with $3^{\circ}$ of movement in each frame. That is, each disk was shifted $3^{\circ}$ in each frame, with the disk at the previous position erased. For no-change trials, the disks retained their original colors throughout the trial. For colorchange trials, the disks' colors were changed at the $24^{\text {th }}$ frame of the motion, and continued with the changed colors until the end of the trial. Two hundred and fifty ms after the completion of the motion sequence, the target dot appeared in the center of either the cued or uncued object. Participants executed a saccade to the object in which the target dot appeared as quickly as possible. The target dot remained on the screen for $1000 \mathrm{~ms}$. By convention, 
we defined the cued and uncued objects in the target display by their spatiotemporal continuity, regardless of their surface feature continuity.

The design of Experiment 1 was 2 (Color Change: No-Change, Change) $\times 2$ (Target Location: Cued Object, Uncued Object). Both factors were within-subjects and there were 72 trials in each condition for a total of 288 test trials. The practice block consisted of 12 trials, and was not included in the analyses. Trial-type was randomized for all subjects.

Data Analysis-Eye tracking data analysis was conducted offline using dedicated software. A velocity criterion (eye rotation $>31 \%$ ) was used to define saccades. The latency of the saccade from the center dot to the target object was the dependent measure in all of the analyses. Trials in which the participant was not at fixation when the target dot appeared or did not make a saccade to the target were excluded from the analysis. After inspection of the latency distribution, latencies faster than $110 \mathrm{~ms}$ were eliminated as likely anticipations ( $2.1 \%$ of the data), and latencies slower than $450 \mathrm{~ms}$ were also eliminated as outliers for saccadic orientation to a simple onset ( $0.5 \%$ of the data). The same saccade latency trimming was calculated for all of the experiments. A total of $21.4 \%$ of the trials were eliminated from the analyses of Experiment 1.

\section{Results and Discussion}

Mean saccade latency data are reported on Figure 2. A 2 (Color Change) $\times 2$ (Target Location) repeated measures ANOVA was conducted to determine whether participants were slower to initiate the saccade to the target when it appeared in the cued object than in the uncued object, and whether color change had an effect on the latencies. The analyses revealed a significant main effect of Target Location, with participants executing significantly slower saccades to the cued object compared to the uncued object, $F(1,19)=$ 15.91, $p=.001$. Neither the main effect of Color Change nor the Color Change $\times$ Target Location interaction were significant $\left(F_{\mathrm{s}}<1\right)$, indicating that color change did not have any effect on the magnitude of IOR.

Separate comparisons showed that participants were slower to execute a saccade to the cued object than the uncued object both when the objects' colors remained the same throughout the trial, $F(1,19)=9.49, p=.006$, and when they were changed to new colors during motion, $F(1,19)=15.04, p=.001$. Specifically, an $8.3 \mathrm{~ms}$ IOR effect was observed for the no-change condition and a $6.5 \mathrm{~ms}$ IOR effect was observed for the color-change condition.

In Experiment 1, the aim was to test whether a salient surface feature discontinuity would disrupt object correspondence. We tested this hypothesis using an object-based IOR paradigm and induced a color change while the objects were moving to new locations. Contrary to what was predicted, the results showed that a salient change in color was not necessarily sufficient to disrupt the object-based IOR effect. In this situation, participants used objects' spatiotemporal continuity to compute correspondence when color information was not reliable.

\section{Experiment 2}

A limitation of Experiment 1 was that on color change trials, color was uninformative with respect to object continuity. That is, the new colors of the objects did not bear any relationship to the objects' original colors. Thus, surface feature information may have been discounted in Experiment 1 because there was often no systematic relationship between the colors before and after motion. In contrast, position information before and after motion was always systematically related given that the objects traveled on smooth paths to the new locations. 
In Experiment 2, we modified the paradigm so that color would be informative with respect to object correspondence and could be used to map the objects before and after motion on every trial. To accomplish this, the objects swapped colors during motion instead of changing to new colors. Therefore, the same colors were present throughout the trial but they were assigned to different objects before and after motion. This created an ambiguity in the target display: on color swap trials, the cued object on the basis of spatiotemporal continuity and the cued object on the basis of surface feature continuity were different objects. Thus, the competition between spatiotemporal and surface feature information in this paradigm affords a comparison of the contributions of both surface feature and spatiotemporal information in computing correspondence. Note that for the sake of consistency, the same- and different-object conditions continue to be defined by spatiotemporal continuity. In this version of the experiment, however, correspondence is ambiguous. The 'same' object for the purpose of IOR could either be the object with continuous spatiotemporal features as the cued object or the object with continuous surface feature (i.e. color) as the cued object.

Again, it was hypothesized that if both surface feature and spatiotemporal information are used in establishing object correspondence, then the magnitude of IOR effect should either be eliminated or reduced when the objects swap colors.

\section{Method}

Participants-Twenty-one undergraduate students from the University of Iowa participated for course credit. All participants had normal, uncorrected vision. Data from two participants were excluded from the analyses for the same reason as in Experiment 1. None of the participants had taken part in Experiment 1.

Stimuli and Apparatus-The same stimuli used in Experiment 1 were used here, with the exception that the disks' colors were randomly selected from the following colors: blue (RGB: 18, 18, 255), green (RGB: 0, 138, 0), and red (RGB: 220, 18, 18). For the color-swap condition, the disks swapped colors at the $24^{\text {th }}$ frame, continued moving with the swapped colors during the last 6 frames of the motion, and remained with the swapped colors until the end of the trial (Figure 1B). The no-swap condition was exactly the same as no-change condition of Experiment 1 (Figure 1A).

Procedure-The procedure was the same as in Experiment 1. A 2 (Color Swap: No-Swap, Swap) $\times 2$ (Target Location: Cued Object, Uncued Object) within-subjects design was used. Similar to Experiment 1, for the purpose of data report, the cued and uncued objects were labeled by their spatiotemporal continuity. There were 288 test trials ( 72 trials in each condition) and 12 practice trials which were not included in the analyses.

Data Analysis-As in Experiment 1, trials in which the participant was not fixating the central fixation when the target dot appeared or did not make a saccade to the target were excluded from the analysis. Latencies faster than $110 \mathrm{~ms}$ (5.2\% of the data) or slower than $450 \mathrm{~ms}$ ( $1.4 \%$ of the data) were also eliminated A total of $24.8 \%$ of the trials were eliminated from the analyses of Experiment 2.

\section{Results and Discussion}

Mean saccade latency data are presented on Figure 3. Saccade latencies were analyzed in a 2 (Color Swap) $\times 2$ (Target Location) repeated measures ANOVA. The analysis showed a significant main effect of Target Location, $F(1,18)=11.69, p=.003$. There was no main effect of Color Swap $(F<1)$. Importantly, the Color Swap and Target Location conditions produced a reliable interaction, $F(1,18)=5.26, p=.034$, with the magnitude of the object- 
based IOR effect in the color-swap condition $(3.8 \mathrm{~ms})$ being significantly smaller than the magnitude of object-based IOR effect in the no-swap condition $(11.8 \mathrm{~ms})$.

Separate comparisons showed that participants were slower to execute a saccade to the cued object relative to the uncued object when there was no color swap $(F(1,18)=14.41, p=$. $001)$, but not when the objects swapped colors $(F(1,18)=1.78, p=.20)$. When both spatiotemporal and surface feature continuity were preserved in the no-swap condition, a significant IOR effect was observed, replicating Experiment 1. However, when the disks swapped colors, this change in surface features disrupted IOR, suggesting that surface feature continuity plays a significant role in defining the objects of objet-based IOR.

In Experiment 2, a significant reduction of object-based IOR was observed when the two objects swapped colors during motion, demonstrating that surface feature consistency plays a significant role in defining object persistence for the purpose of IOR. We consider two plausible explanations for this effect. They need not be mutually exclusive. First, a salient change in surface features might have disrupted the computation of object correspondence, eliminating the mapping of objects across motion and thus eliminating object-based IOR. Second, inhibitory tags might be associated independently with the spatiotemporal features and the surface features of an object. In other words, a previously attended color value may be inhibited independently of the location where this color appears ${ }^{3}$, and the position of the previously attended object (updated with motion) may be inhibited independently of the surface features present at that location. If so, then in the Experiment 2 color-swap trials, inhibition of the surface features and spatiotemporal features of the attended object might have offset each other, as they were associated with different locations in the target display.

\section{Experiment 3}

The results from Experiment 2 suggest that when the same surface feature values are present before and after motion, surface feature information is used to compute object correspondence. In Experiment 2 (and in Experiment 1), spatiotemporal information was continuous and thus informative. To investigate whether color continuity alone can support an object-based IOR, the paradigm was again modified to eliminate spatiotemporal continuity. Linking motion between the original and new locations was removed; the objects disappeared from their original locations, and after a blank ISI, appeared abruptly at their new locations. This procedure resulted in spatial ambiguity, because the objects' new locations were equidistant from each of their original locations (Mitroff \& Alvarez, 2007). The only information the participants could use for determining the cued and uncued object was surface features (i.e. colors).

The presence or absence of object-based IOR in this method can help to disentangle the possible explanations for the color-swap effect in Experiment 2. The first possibility is that a discontinuity in an object's features can, if sufficiently salient, disrupt the computation of object persistence, eliminating object-based IOR. If spatiotemporal and surface feature information are in conflict, the presence of IOR therefore depends on whether consistency on one dimension is capable of overcoming the absence of consistency on the other. In Experiment 1, spatiotemporal consistency was sufficient to establish correspondence despite the fact that the objects changed to new color values. In Experiment 3, the presence/absence of object-based IOR will indicate whether surface feature consistency is sufficient to establish correspondence despite the fact that the objects change to new locations.

\footnotetext{
${ }^{3}$ There is some evidence indicating inhibition associated with color. Law, Pratt, and Abrams (1995) measured IOR with a color discrimination task, and showed that participants were slower to detect a colored object when its color matched a previously attended color. An important limitation of that study, however, is that both cued and uncued object were presented at the center of the screen. Therefore, this type of inhibition is not object-based, and is not directly related to the paradigm used in the present study.
} 
The second possibility is that inhibition is directly associated with particular color values and with particular spatiotemporal features, in which case surface feature and location inhibition would be established by separate inhibitory tags. In Experiment 3, there should be no inhibition associated with the new locations of the objects as there is no position continuity. Color-based inhibition should still be functional, however, as the same color values are present in the preview and target displays, and IOR should be found at the location of the previously attended color value. This contrasts with the design of Experiment 2 , in which inhibition associated with spatiotemporal and surface features potentially counteracted each other. Now, only surface feature inhibition should be operational, and thus a significant delay in orienting to the previously attended color should be observed.

In sum, the "disruption of object correspondence" account holds that color continuity will support object-based IOR only if the match is sufficiently strong to overcome the presence of a spatiotemporal discontinuity. The "separate inhibitory tags" account predicts that color continuity should generate IOR in a manner that is largely independent of spatiotemporal continuity, as the inhibitory tag associated with the previously attended color is separate from the tag associated with the previously attended location.

\section{Method}

Participants-Twenty-one undergraduate students from the University of Iowa participated in this experiment for course credit. Data from 6 participants were excluded for the same reason as that described in Experiment $1 .{ }^{4}$ None of the participants had taken part in previous experiments.

Stimuli and Apparatus-The same stimuli as in Experiment 2 were used in this experiment. In all trials, the disks had different colors, randomly chosen from three colors used in Experiment 2.

Procedure-The procedure was the same as in Experiment 2 except that there was no linking motion. After the peripheral and central cueing, the disks disappeared for $250 \mathrm{~ms}$, and then reappeared $90^{\circ}$ (in polar coordinates) away from their initial positions (either clockwise or counterclockwise). As in the previous experiments, the target dot appeared on either the cued or uncued disk after a 250 ms delay.

Unlike Experiments 1 and 2, there was now only one factor: Target location (Cued Color, Uncued Color).

Data Analysis-As in the previous experiments, trials on which the participant was not fixating the central fixation when the target dot appeared or did not make a saccade to the target were excluded from the analysis. Latencies faster than $110 \mathrm{~ms}(4.0 \%$ of the data) or slower than $450 \mathrm{~ms}$ ( $2.6 \%$ of the data) were also eliminated A total of $32.1 \%$ of the trials were eliminated from the analyses of Experiment 3.

\section{Results and Discussion}

Because there was no linking motion, the only information participants could use for object correspondence was the colors of the disks. Therefore, the magnitude of IOR was calculated as the difference in saccade latencies to the objects with the cued and uncued colors.

\footnotetext{
${ }^{4}$ The same analyses were also run on data from all participants. No significant difference between cued and uncued color objects was found $(F(1,20)=.450, p=.51)$.
} 
The saccade latency data is presented on Figure 4. There was no significant difference between saccade latencies to the target dot when it appeared in an object with the cued and uncued color, $F(1,14)=2.74, p=.12$. This result suggests that color information alone is not sufficient to establish object correspondence, at least in the present paradigm.

Experiment 3 was designed to test the possibility that surface feature information alone can be used to compute object correspondence. No significant object-based IOR effect was observed, suggesting that color was not sufficient in this experiment to establish correspondence in the presence of a salient spatiotemporal discontinuity. This result is potentially difficult to reconcile with the idea that color and location receive separate inhibitory tags. The only possible source of inhibition in Experiment 3 was color-based, and if the previously attended color value had been tagged for inhibition, IOR should have been observed. The results are therefore consistent with the hypothesis that both spatiotemporal and surface feature information contribute to defining the persisting objects of object-based IOR.

There is one circumstance in which the Experiment 3 results might still be consistent with a "separate inhibitory tags" account. It is possible that the removal of the two objects before the change in position might have been perceived as removal of the entire scene, "resetting" the inhibitory tags that had been assigned to the colors. Such an account is broadly consistent with evidence that scene removal eliminates IOR (Takeda \& Yagi, 2000; Müller \& von Mühlenen, 2000; Klein \& MacInnes, 1999). Thus, we cannot discount entirely the possibility that color received independent inhibitory tags. However, one reason to be cautious in endorsing this alternative is that the fixation cross remained visible even when the objects were removed, providing some scene-level continuity across the trial. A stronger test of this possibility would be to include additional static, contextual structure to the display so that the removal of the object stimuli could not be plausibly interpreted as a change in the scene as a whole.

\section{General Discussion}

In the present study, we investigated the contributions of spatiotemporal and surface feature information to object correspondence across three experiments. Object-based IOR provided a measure of object correspondence. The spatiotemporal and/or surface feature properties of the objects were manipulated. In Experiment 1, significant object-based IOR was observed both when the objects retained their colors throughout the trial and also when they changed their colors while moving to new locations ${ }^{5}$. In Experiment 2, IOR was eliminated when the objects swapped colors during motion. In Experiment 3, color information alone was not sufficient to establish object correspondence in the presence of a salient spatiotemporal discontinuity.

These results indicate that both spatiotemporal and surface feature information contribute to defining the persisting objects of object-based IOR. In Experiment 2, object correspondence could have been established solely on the basis of spatiotemporal properties of the objects; each object moved on a smooth and continuous path to its new location. Thus, the finding of a surface feature effect on IOR is inconsistent both with the view that object correspondence

\footnotetext{
${ }^{5}$ Although we found no significant effect of color change in Experiment 1, Moore and Enns (2004) found that a salient change in color disrupts object continuity in an apparent motion paradigm. There are at least two significant differences between the apparent motion and object-based IOR paradigms. First, in their apparent motion paradigm, there is only one object in the display, and participants' attention is allocated to that object throughout the trial. Therefore, a color change may be more noticeable because the object is in the focus of attention. However, in our object-based IOR paradigm, there were two objects in the display which could have resulted in a broader distribution of attention or dividing attention between two objects. In that case, the disruptive influence of color change may have been weakened.
} 
operations consult only spatiotemporal features (Kahneman et al., 1992) and with the view that surface features are consulted only when spatiotemporal information is absent or ambiguous (Flombaum et al., 2009; Scholl, 2007). Instead, the results are consistent with a growing body of evidence that surface feature continuity plays a significant role in computing online object correspondence across the brief episodes of change and disruption that characterize real-world vision (Feldman \& Tremoulet, 2006; Hollingworth \& Franconeri, 2009; Hollingworth et al., 2008; Moore \& Enns, 2004; Moore et al., 2007; Moore et al., 2010; Richard et al., 2008).

Although the results of Experiment 2 demonstrated a role of surface feature continuity in establishing object-based IOR, there was an asymmetry between the color and spatiotemporal results in Experiments 1 and 3. Specifically, the results of Experiment 1 suggest that spatiotemporal information was sufficient to establish object correspondence in the presence new color values in the display. In contrast, the results of Experiment 3 showed that surface feature information was not sufficient to establish correspondence in the presence of new position information in the display. The asymmetry is consistent with a recent study of static IOR (Hilchey, Ivanoff, Taylor, \& Klein, 2011) in which position information was found to provide a greater contribution to IOR effects than identity information. However, comparison of the relative contributions of spatiotemporal and surface features to object-based IOR is complicated by difficulties in equating the salience of a particular change in each domain. That is, one would need to control or manipulate the low-level salience of, for example, a change in color versus a change in location. Such cross-dimensional assessments of low-level change are exceedingly difficult. It might be the case that the location change in Experiment 3 was simply more perceptually salient than the change in a single surface feature attribute, color, in Experiment 1: Although the object's color changed, many of the surface feature properties of the objects did not (e.g., size, shape, and texture). Therefore, changes in multiple surface features, like color and shape, may make the change perceptually more salient, and increase the effect of surface features on object correspondence. Thus, we can conclude that both surface features and spatiotemporal features contribute to object-based IOR, but it would be premature to draw strong conclusions about the relative importance of the two sources of information.

\section{Acknowledgments}

We would like to thank Ray Klein, Steve Mitroff, and an anonymous reviewer for their helpful comments on earlier draft of this manuscript.

\section{References}

Abrams RA, Dobkin RS. Inhibition of return: Effects of attentional cuing on eye movement latencies. Journal of Experimental Psychology: Human Perception and Performance. 1994; 20(3):467-477. [PubMed: 8027710]

Burke L. On the tunnel effect. Quarterly Journal of Experimental Psychology. 1952; 4:121-138.

Feldman J, Tremoulet PD. Individuation of visual objects over time. Cognition. 2006; 99:131-165. [PubMed: 16545625]

Flombaum, JI.; Scholl, BJ.; Santos, LR. Spatiotemporal priority as a fundamental principle of object persistence. In: Hood, B.; Santos, L., editors. The origins of object knowledge. Oxford University Press; 2009. p. 135-164.

Hilchey MD, Ivanoff J, Taylor TL, Klein RM. Visualizing the temporal dynamics of spatial information processing responsible for the Simon effect and its amplification by inhibition of return. Acta Psychologica. 2011; 136:235-244. [PubMed: 20932500]

Hollingworth A, Franconeri SL. Object correspondence across brief occlusion is established on the basis of both spatiotemporal and surface feature cues. Cognition. 2009; 113:150-166. [PubMed: 19729155] 
Hollingworth A, Richard AM, Luck SJ. Understanding the function of visual short-term memory: Transsaccadic memory, object correspondence, and gaze correction. Journal of Experimental Psychology: General. 2008; 137:163-181. [PubMed: 18248135]

Kahneman D, Treisman A, Gibbs BJ. The reviewing of object-files: Object-specific integration of information. Cognitive Psychology. 1992; 24:175-219. [PubMed: 1582172]

Klein RM, MacInnes WJ. Inhibition of return is a foraging facilitator in visual search. Psychological Science. 1999; 10(4):346-352.

Law MB, Pratt J, Abrams RA. Color-based inhibition of return. Perception \& Psychophysics. 1995; 57(3):402-408. [PubMed: 7770330]

Mitroff SR, Alvarez GA. Space and time, not surface features, guide object correspondence. Psychonomic Bulletin \& Review. 2007; 14(6):1199-1204. [PubMed: 18229497]

Mitroff SR, Scholl BJ, Wynn K. The relationship between object files and conscious perception. Cognition. 2005; 96:67-92. [PubMed: 15833307]

Moore CM, Enns JT. Object updating and the flash-lag effect. Psychological Science. 2004; 15(12): 866-871. [PubMed: 15563333]

Moore CM, Mordkoff JT, Enns JT. The path of least persistence: Object status mediates visual updating. Vision Research. 2007; 47:1624-1630. [PubMed: 17451777]

Moore CM, Stephens T, Hein E. Features, as well as space and time, guide object persistence. Psychonomic Bulletin \& Review. 2010; 17:731-736. [PubMed: 21037174]

Morgan HM, Tipper SP. Shape specific inhibition of return. European Journal of Cognitive Psychology. 2007; 19(3):321-334.

Müller HJ, von Mühlenen A. Probing distractor inhibition in visual search: Inhibition of return. Journal of Experimental Psychology: Human Perception and Performance. 2000; 26(5):1591-1605. [PubMed: 11039487]

Noles NS, Scholl BJ, Mitroff SR. The persistence of object file representations. Perception \& Psychophysics. 2005; 67(2):324-334. [PubMed: 15973783]

Hein, E.; Moore, CM. Do surface features help? How the visual system disambiguates ambiguous motion. Poster presented at the 9th Annual Meeting of the Vision Sciences Society; Naples, Florida. 2009 May.

Paul MA, Tipper SP. Object-based representations facilitate memory for inhibitory processes. Experimental Brain Research. 2003; 148:283-289.

Posner, MI.; Cohen, Y. Components of visual orienting. In: Bouma, H.; Bouwhuis, D., editors. Attention and performance X. London: Erlbaum; 1984. p. 531-556.

Pylyshyn ZW. Situating vision in the world. Trends in Cognitive Sciences. 2000; 4:197-207. [PubMed: 10782106]

Richard AM, Luck SJ, Hollingworth A. Establishing object correspondence across eye movements: Flexible use of spatiotemporal and surface feature information. Cognition. 2008; 109:66-88. [PubMed: 18760406]

Scholl BJ. Object persistence in philosophy and psychology. Mind \& Language. 2007; 22(5):563-591.

Takeda Y, Yagi A. Inhibitory tagging in visual search can be found if search stimuli remain visible. Perception \& Psychophysics. 2000; 62(5):927-934. [PubMed: 10997039]

Taylor TL, Klein RM. Visual and motor effects in inhibition of return. Journal of Experimental Psychology: Human Perception and Performance. 2000; 26:1639-1656. [PubMed: 11039490]

Tipper SP, Weaver B, Jerreat LM, Burak AL. Object-based and environment-based inhibition of return of visual attention. Journal of Experimental Psychology: Human Perception and Performance. 1994; 20(3):478-499. [PubMed: 8027711] 


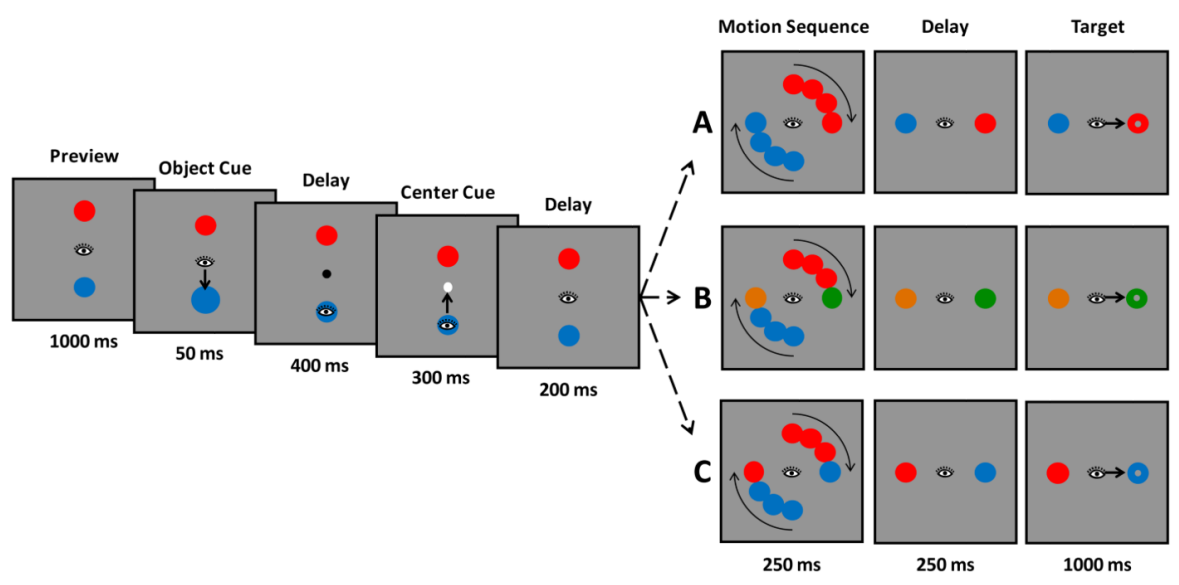

Figure 1.

The sequence of events for the three main conditions used in Experiments 1 and 2. A. No change condition (Experiments 1 and 2). The two disks retained their original colors throughout the motion sequence. B. Color-change condition (Experiment 1). The two disks changed to new colors near the end of the motion sequence. C. Color-swap condition (Experiment 2). The two disks exchanged colors near the end of the motion sequence. The stimuli depicted in this figure are not always to scale. In addition, the motion sequence shows stimuli at multiple locations to illustrate the motion path and the color changes. In the experiments, each of the two disks was presented in only one location in each frame of the motion sequence. 


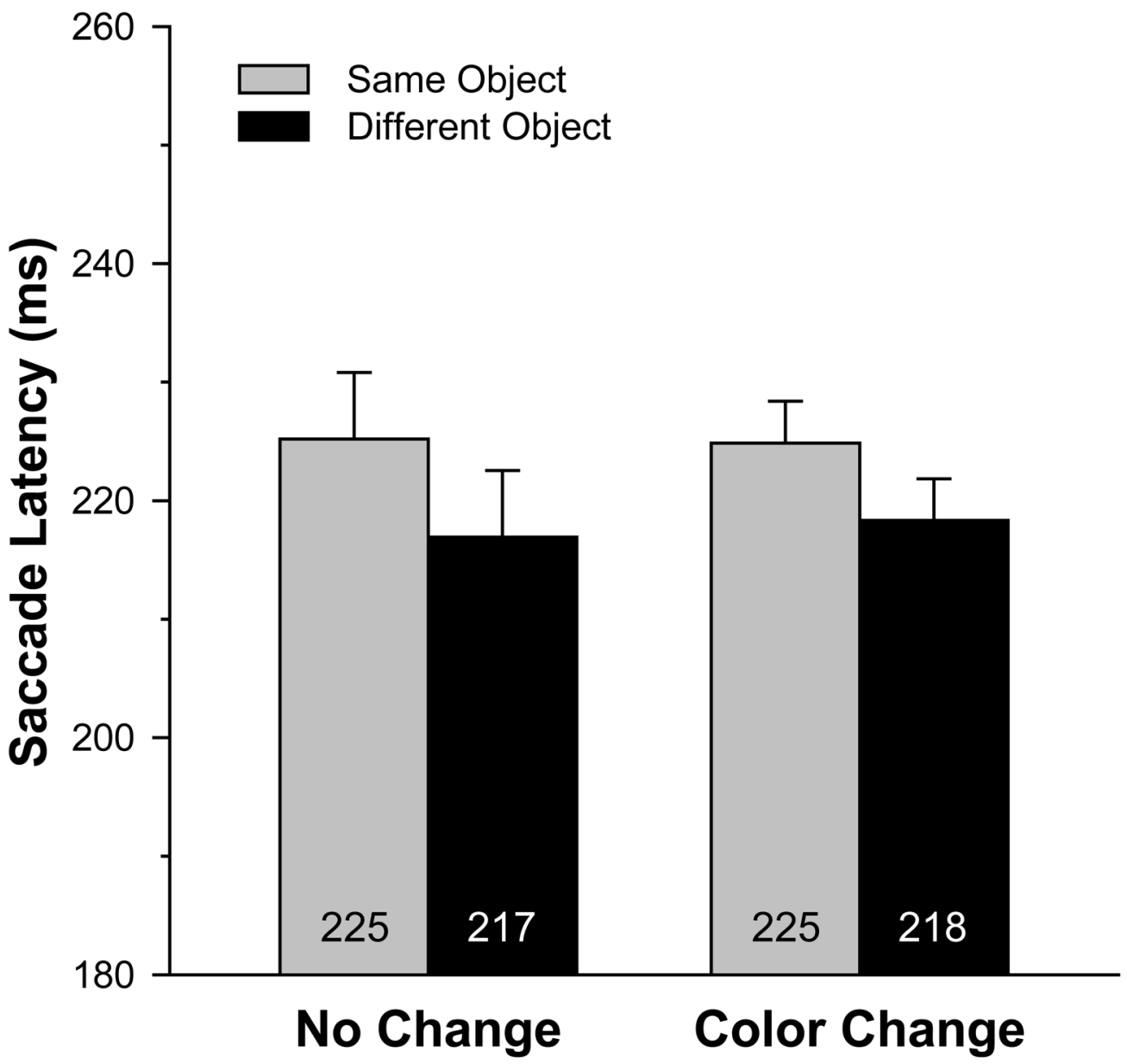

Figure 2.

Mean saccade latency plotted as a function of color-change conditions of Experiment 1. In each data figure, error bars are $95 \%$ within-subject confidence intervals based on the error term of the object effect in each of the color-change conditions. 


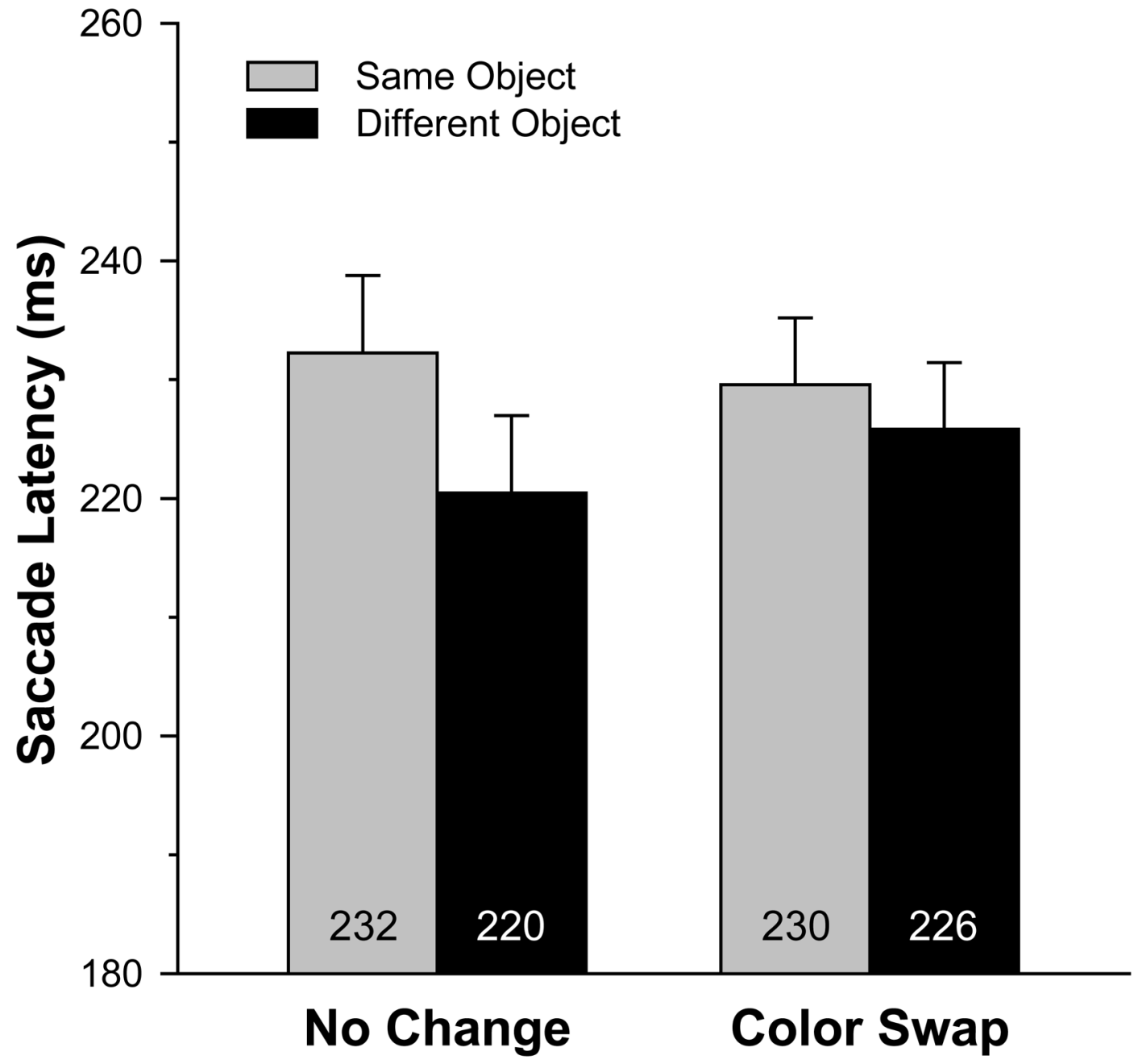

Figure 3.

Mean saccade latency plotted as a function of color-swap conditions of Experiment 2. 


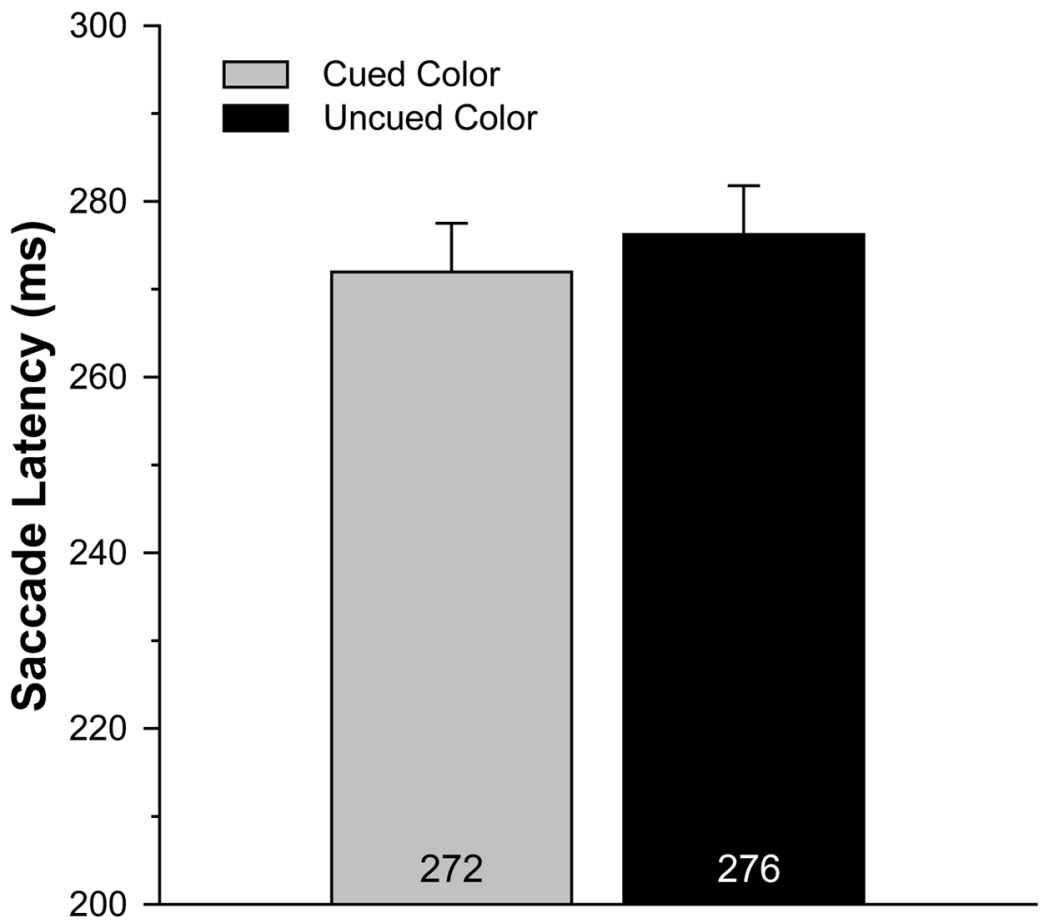

Figure 4.

Mean saccade latency for same/different color objects in Experiment 3. 